Results On re-evaluation of HRCT $(\mathrm{n}=69)$, eight scans had no evidence of ILD. 49 scans had evidence of ILD (NSIP = 41; UIP $=5$; non-specific pattern of ILD $=3$ ). Following comparison with the initial reporting radiologists' reports, twelve patients, with no previous diagnosis of ILD, were identified with the NSIP phenotype. The autoantibody model, using positive ACA and anti-Scl70 status, correctly classified $64.5 \%$ of cases overall $(\mathrm{n}=62 ; \mathrm{p}=0.05)$.

Conclusions The role of objective HRCT evaluation, by a specialist radiologist, is superior in the detection of ILD, even in the ubiquitous NSIP phenotype, when compared to general radiology review and predictive autoantibody profiling of the same patients.

\section{P32 ROLE OF NON ACID AND PROXIMAL REFLUX IN SCLERODERMA-ASSOCIATED INTERSTITIAL LUNG DISEASE}

${ }^{1} \mathrm{~A}$ De Lauretis, ${ }^{1} \mathrm{~S}$ Ward, ${ }^{2} \mathrm{C}$ Murray, ${ }^{2} \mathrm{C}$ Clayman, ${ }^{3} \mathrm{~V}$ Ong, ${ }^{3} \mathrm{C}$ Denton, ${ }^{1} \mathrm{~A}$ Bikov, ${ }^{1} \mathrm{D}$ Visca, ${ }^{1} \mathrm{G}$ Lindahl, ${ }^{4} \mathrm{~A}$ Chetta, ${ }^{4} \mathrm{M}$ Aiello, ${ }^{5} \mathrm{~W}$ Wuyts, ${ }^{6} \mathrm{M}$ Kreuter, ${ }^{1} \mathrm{~T}$ Maher, ${ }^{1} \mathrm{C}$ Stock, ${ }^{1} \mathrm{AU}$ Wells, ${ }^{1} \mathrm{E}$ Renzoni. 'Interstitial Lung Disease Unit, Royal Brompton Hospital, London, UK; ${ }^{2}$ Department of Gastroenterology, Royal Free Hospital, London, UK; ${ }^{3}$ Department of Rheumatology, Royal Free Hospital, London, UK; ${ }^{4}$ Department of Respiratory Medicine, University of Parma, Parma, Italy; ${ }^{5}$ Department of Respiratory Medicine, University Hospitals Leuven, Leuven, Belgium; ${ }^{6}$ Department of Respiratory Medicine, University of Heidelberg, Heidelberg, Germany

\subsection{6/thoraxjnl-2015-207770.169}

Background Oesophageal involvement is extremely common in patients with scleroderma. This prospective observational study (NCT02136394) addresses the relationship between gastro-oesophageal reflux (GORD) and scleroderma-associated interstitial lung disease (SSc-ILD), and evaluates the clinical utility of noninvasive tests of microaspiration.

Materials and methods We present preliminary results of the first 27 enrolled patients (median age 59 [ $\mathrm{min} / \mathrm{max} 35 / 79$ ], median $\mathrm{FVC}=74 \%$ [38/128\%], median DLCO $=39 \%[21 /$ $72 \%$ ], female $70 \%$, diffuse SSc 33\%). Collected clinical data included $24 \mathrm{hr}$ impedance (carried out off PPI), respiratory (KBILD and Leicester cough questionnaires) and GORD symptom questionnaires (UCLA SCTC GIT 2.0 Questionnaire, Reflux Disease Questionnaire RDQ), as well as full lung function test data. Pepsin levels were measured in saliva in all patients, and in a subset of 6 patients in bronchoalveolar lavage (BAL).

Results Non acid reflux and proximal reflux were detected in $54 \%$ and $49 \%$ of patients, respectively. In the subgroup of patients with normal DeMeester score (i.e. global impedance index of acid exposure), 66\% had non acid reflux episodes. The DeMeester score (median 14.2 [min/max 0.8/156]) was correlated with total scores GORD questionnaire scores (e.g. RDQ, $r$ $=0.68 \mathrm{p}=0.003$; GIT 2.0, $\mathrm{r}=0.68 \mathrm{p}=0.004)$, but not with K-BILD, Leicester questionnaire, or saliva pepsin. Proximal reflux episodes were moderately correlated with the Leicester total score $(\mathrm{r}=-0.76 \mathrm{p}=0.002)$ and with saliva pepsin $(\mathrm{r}=$ $0.46 \mathrm{p}=0.05$ ). Saliva pepsin (median concentration $2.34 \mathrm{ng} / \mathrm{ml}$ [2.34/12.4]) was correlated with the impedance cough index association $(\mathrm{r}=0.53, \mathrm{p}=0.02)$. BAL pepsin was present in all six cases (median concentration $2.34 \mathrm{ng} / \mathrm{ml}[2.34 / 12.4]$ ) and was correlated with FVC $(\mathrm{r}=-0.8, \mathrm{p}=0.04)$. Lung function test parameters were not correlated with saliva pepsin, but were significantly, if loosely, correlated with impedance measures of acid exposure in the recumbent position (e.g.\% time of exposure, $r$ $=-0.43 \mathrm{p}=0.04)$.
Conclusions Proximal and non acid reflux are highly prevalent in the SSc-ILD population and are associated with a high symptom burden. Pepsin is measurable in BAL of SSc-ILD patients and suggests microaspiration into the lungs, although larger numbers are needed to confirm these findings and define whether saliva pepsin measurement could represent a useful non invasive marker of microaspiration.

\section{P33 RITUXIMAB AS RESCUE THERAPY IN ADVANCED PROGRESSIVE SYSTEMIC SCLEROSIS ASSOCIATED INTERSTITIAL LUNG DISEASE}

M Kokosi, P Saunders, K Karagiannis, F Chua, TM Maher, EA Renzoni, AU Wells. Royal Brompton Hospital, London, UK

\subsection{6/thoraxjnl-2015-207770.170}

Introduction Severe interstitial lung disease associated with systemic sclerosis (SSc-ILD) often has an inexorably progressive course. Prevention or retardation of disease progression (as seen in both SLS and the FAST trials) is the only realistic treatment goal in most cases. Rituximab is a B- lymphocyte depleting monoclonal antibody which has proven efficacy in a spectrum of treatment-refractory interstitial lung diseases. Data on the impact of rituximab therapy on SSc-ILD outcomes are limited.

Methods 18 patients with severe progressive SSc-ILD, despite conventional immunosuppression, were studied retrospectively. Serial change in FVC and DLco was quantified as percentage relative change from baseline, Pulmonary function trends pre (3-17 months) and post (3-11 months) Rituximab therapy were compared using paired t-testing.

Results 18 patients (four male), with a median age 57.5 ( \pm 15.9$)$ received treatment with rituximab between 2012 and 2014. The median follow-up period was 7.96 (range 3.1-11.2) months. One patient died from heart failure. Rituximab was well tolerated. At the time of rituximab treatment, patients had severe pulmonary function impairment (median FVC 50.5\%, range 3684\%; median DLco 25\%, range 14-41\%). On paired testing, there was a reduction in serial FVC decline following Rituximab therapy $(-10.1 \% \pm 7.8 \%$ versus $-1.5 \% \pm 8.7 \%, \mathrm{p}=0.01)$ and $\mathrm{a}$ similar reduction in serial DLco decline $(-15.6 \% \pm 16.8 \%$ vs. $1.0 \% \pm 27.2 \%, \mathrm{p}=0.05)$.

Conclusion The addition of Rituximab was associated with a significant reduction in serial pulmonary function decline in patients with advanced progressive SSc-ILD, not controlled by intense conventional immunomodulation. These findings provide further support for the use of Rituximab as rescue therapy in severe SSc-ILD.

\section{P34 SARCOIDOSIS AND CO-EXISTENT ASPERGILLUS LUNG DISEASE}

S Gudur, E Nuttall, C Harris, N Chaudhuri, C Leonard, E Muldoon. University Hospital of South Manchester, Manchester, UK

\subsection{6/thoraxjnl-2015-207770.171}

Sarcoidosis is a multisystem disorder which affects the lungs and in a small percentage of cases may result in fibrosis and cystic cavitating lesions. Chronic pulmonary aspergillosis (CPA) typically affects patients with underlying lung conditions; immunosuppressive therapy is not recommended due to risk of progression or spread of Aspergillus infection. Sarcoid patients 
are often significantly debilitated when they develop co-existent Aspergillus lung disease. They can present with worsening breathlessness, weight loss and significant haemoptysis. There is no consensus on how to best treat patients diagnosed with Sarcoid and CPA.

Methods A retrospective review of patients diagnosed with Sarcoidosis and CPA was performed. Cases were identified from a database held of patients diagnosed with CPA group in our specialist clinic. Patient demographics, presentation, laboratory parameters, radiology results were recorded and data analysed.

Results 38 patients with sarcoidosis were diagnosed with CPA from 2009 - March 2015. 23/36 (63\%) were male. 14/36 (38\%) patients were affected by breathlessness with MRC dyspnoea score $\geq 3.15 / 36(41 \%)$ of patients were on maintenance Prednisolone between $5-10 \mathrm{mg} /$ day and 3 patients on other steroid sparing agents. 12/28 patients had obstructive spirometry and average predicted gas transfer factor at 49\% (range $20-75 \%$ ).

$33 / 36$ (91\%) had CPA defined by presence of serum precipitins and $50 \%$ of patients had aspergillomas detected in the cavities on radiology. $27 / 36(75 \%)$ of patients were on antifungal therapy. On regular follow-up, antifungal therapy was titrated based on serum azole levels and as tolerated.

4/36 (11\%) patients had been treated for proven TB and 2/36 were on empirical treatment until $\mathrm{TB}$ diagnosis was excluded when there was clinical and radiological deterioration.

Conclusions Although sarcoidosis has slight female predominance, in this cohort of patients with CPA and sarcoid 63\% were males. Many of the patients had significant lung disease with high MRC dyspnoea scores, and obstructive spriometry often with low gas transfers. The management of CPA in the setting of sarcoidosis is complex due to the risk of immunosuppression and interactions with antifungal therapy. Close working between specialties is important in optimising such patients, to ensure the best patient outcomes.

\section{P35 IDENTIFYING NOVEL PREDICTORS OF OUTCOME IN SARCOIDOSIS}

P Minnis, M Poland, G Nolan, SC Donnelly. Department of Respiratory Medicine, Trinity Centre for Health Sciences, Tallaght Hospital, Dublin, Ireland

\subsection{6/thoraxjnl-2015-207770.172}

Background and objectives Sarcoidosis is a multisystem disease of unknown aetiology characterised by a Th1 granulomatous immune response. Predicting need for treatment in sarcoidosis is based on well-recognised prognostic indicators. ${ }^{1}$ We aimed to interrogate a large cohort of sarcoidosis patients in order to identify novel predictors for treatment and disease progression defined physiologically and radiologically.

Methods Clinical data from 338 patients with sarcoidosis was recorded retrospectively including; age at diagnosis, gender, Scadding CXR stage at presentation, presence of Erythema Nodosum (EN), baseline inflammatory markers, immunoglobulin levels, skin prick testing (SPT), pulmonary function tests, BAL results and vitamin D levels. There was a minimum follow up of 2 years for all patients. The primary outcome measure was the need for treatment, defined as requiring oral corticosteroids for 6 months or more. Secondary outcome measures included need for steroid sparing agents and progression defined radiologically using scadding criteria or HRCT when available and assessing pulmonary function test decline.
Results Multivariate analysis and modelling of all presenting features was performed. Patients were characterised into four groups-limited no treatment, limited requiring steroids, chronic but stable and chronic progressive steroid dependent. Overall the treatment rate was $50.4 \%$ in this cohort. In the chronic cohort 32\% required steroid sparing agents. Initial analysis confirmed previously known prognostic indicators including Scadding CXR stage, age $>40$ and presence of EN OR 0.24 (0.13$0.45), \mathrm{p}<0.0001$ at diagnosis. Additional predictors of prognosis included IgG levels, positive skin prick testing and vitamin D levels. In the group with elevated levels of IgG (IQR 15.7-19.8) at assessment the treatment rate was $77.1 \%$ OR 3.3 (1.5 to 7.6) $\mathrm{p}$ value 0.004 . In the group with positive SPT, only $17.4 \%$ required treatment, OR $0.21(0.07-0.62)$, p value 0.005 .

Conclusions These results indicate possible new markers of prognosis in terms of treatment and disease progression in sarcoidosis. Phenotyping sarcoid patients in this way may be valuable in designing future clinical trials in sarcoidosis.

\section{REFERENCE}

1 Neville E, Walker AN, Geraint James D. Prognostic factors predicting the outcome of sarcoidosis: an analysis of 818 patients. QJM 1983;4:525-533

\section{P36 ETHNIC DIFFERENCES IN COMPOSITE PHYSIOLOGIC INDEX (CPI) IN PULMONARY SARCOIDOSIS: A 10-YEAR EXPERIENCE IN A SPECIALIST SARCOIDOSIS CLINIC}

${ }^{1} \mathrm{~T}$ Tully, ${ }^{1} \mathrm{~J}$ Galloway, ${ }^{2} \mathrm{~J}$ Lally, ${ }^{1} \mathrm{E}$ Silber, ${ }^{1} \mathrm{P}$ Brex, ${ }^{1} \mathrm{~S}$ Walsh, ${ }^{1} \mathrm{G}$ Larkin, ${ }^{1} \mathrm{~J}$ Barker, ${ }^{1} \mathrm{~S}$ Birring. ${ }^{1}$ Kings College Hospital NHS Foundation Trust, London, UK; ${ }^{2}$ Kings College London, London, UK

\subsection{6/thoraxjnl-2015-207770.173}

Introduction and objectives CPI has been reported to be an accurate predictor of mortality in sarcoidosis and IPF. A CPI score of $>40$ is associated with a 5 year survival probability of $50 \% .{ }^{1}$ There is a paucity of studies of CPI in patients of black ethnicity. We investigated CPI in a large cohort of patients attending a specialist sarcoidosis clinic serving a large African Caribbean community in South-East London.

Methods 503 subjects with a diagnosis of sarcoidosis were assessed between May 2005 and May 2015. Relevant data needed to calculate CPI and ethnicity information was available in 297 patients with pulmonary involvement (based on radiological evidence $+/$ - histological evidence). We calculated CPI with the following formula: $91.0-(0.65 \times$ percent predicted DLCO $)$ - $(0.53 \times$ percent predicted FVC $)+(0.34 \times$ percent predicted FEV1). Clinical demographics, organ involvement, lung function, histology and subsequent therapy were systematically recorded (Table 1).

Results There were significant differences in CPI between all ethnic groups (ANOVA, p $<0.001$ ). Black patients had a higher mean CPI score compared to white patients (39.0 (14.2) vs 29.9 (18.5), $\mathrm{t}=-4.129, \mathrm{p}<0.001$ ) (Table 1 ). CPI was significantly higher in black Caribbean patients versus black African patients (41.2 (14.4) vs 36.3 (13.7), $\mathrm{t}=2.656 \mathrm{p}=0.008)$. CPI was weakly correlated with number of immunosuppressant medications used; correlation coefficient $\mathrm{r}=0.214, \mathrm{p}<0.001$. CPI was not associated with age at presentation, gender, initial requirement of treatment or prediction of $\geq 2$-organ involvement.

Conclusion Black patients have more severe disease than other ethnicities, with the most severe illness in those of black Caribbean ethnicity, who have the worst CPI scores. Further studies 